\title{
Author Index Vol. 66,1994
}

Abstracts of the 5th European Workshop on Neonatology published in No. 2-3 have their own Author Index

Adamson, S.L. 339 Akyol,Ö. 267 Albers,S. 182 Alvaro,R. 188 Amato,M.M. 288 Andiné,P. 205 Arad,I. 71

Bancalari, E. 137 Bandstra,E. 137 Bendaoud,B. 175 Bengtsson, P.-O. 230 Berry, CJ. 112 Berry, S.M. 56 Birtles,M.J. 280 Bishai,I. 339 Bratlid,D. 238 Bräunlich,H. 352 Breier,B.H. 280

Broughton Pipkin, F. 22

Case,C.L. 330 Cashore,WJ. 288 Cates,D. 188 Chevalier, R.L. 294 Chik,L. 56 Childress, S. 214 demons, G.K. 50 Coceani, F. 339 Contant,C.F. 359 Covert, R.F. 316 Cremades, A. 119

Dani, C. 45

Delivoria-Papadopoulos, M. 367 DelToro,J. 359 deMello,D.E. 76 Devaskar, U.P. 76 Diekmann, L. 10 Diemer,N.-H. 205 Dilmen,U. 267 Dobbie, P.M. 112 Dombrowski, M.P. 56 Donnou,M.D. 175 Doty,S.E. 33 Drummond, W.H. 316

Duara,S. 137 Duke,J.C. 247 Durak,I. 267

El-Mohandes, A.A.E. 195 Engelberts, D. 339 Ergaz, Z. 71 Eriksson, U.J. 230

Farstad,T. 238

Galindo,J.D. 119 Gelardi,N.L. 288 Geldern, T. von 294 Gilland,E. 205 Gillette, P.C. 330

Gluckman, P.D. 280 Goddard-Finegold, J. 359 Gorostiza, P. 221 Govindrajan, R. 76 Green, M. 1 Guarner, V. 221 Guillois,B. 175

Hagberg, H. 205 Hammerman, C. 128 Hartley, R. 1 Hernandez, E.H. 221 Hetmanski,D. 22

Hewett,K, W. 330 Heydeck,D. 106 Heyman, S. 76 Huerto, R. 221 Huffme, M.E. 214 Hurd,W.W. 56 Huter,O. 272

Jefferies, A.L. 86 Jones, S.A. 339 Jorch,G. 182

Kalhoff,H. 10 Kashyap, S. 65 Katona, I.M. 195 Kaya,I.S. 267 Khashaba, M.T. 146

Kiessling, C. 16 Kilguss,N.V. 288 Küiç,Z. 267 Kitapçi,F. 267 Knoppert, D.C. 311 Kunz,C. 10

Lawson, A. 247 Lee,D.S.C. 311 Levene, M.I. 1 Louis, P.T. 359 Lun,A. 106

McCormack, W.D. 33 McGowan, J.C. Ill 367 McGowan, J.E. 367 Machado, C.S.M. 324 Maffei, H.V.L. 324 Malicdem, M. 76 Manz,F. 10 Mayr,A. 272 Meister,B. 272 Mellor,DJ. 280 Michael, L.H. 359 Mills, S. 188 Mishra, O.P. 367 Moreno, S.J. 221 Morikawa,Y. 100

Norling,L.L. 294 Norton, J.L. 339

Obladen,M. 16 Odden,J.-P. 238 Ohishi,I. 100 Okada,T. 100

Papies,B. 106 Parker, M.D. 247 Peñafiel,R. 119 Pezzullo, J.C. 50 Pueschel, S.M. 50

Quinn,M.W. 1

377

Rey,M. 16 Rigatto,H. 188 Rivas,R.A. 195 Robinson, G.M. 112 Rodrigues, M.A.M. 324

Roigas,C. 106 Roigas,J. 106 Rozycki,HJ. 93 Rubaltelli, F.F. 45 Rushforth, J.A. 1

Saleh,A 1.56 Sánchez-Capelo, A. 119 Schmidt, M. 352 Seagrave, R.C. 33 Segerer, H. 16

Seidler,FJ. 254 Sekar,K,C. 247 Senses, D.A. 267 Shimada,M. 302 Sikes,J. 214 
Sizun,J. 175 Slotkin,T.A. 254 Sokol,R.J. 56 Spencer, G.S.G. 112 Sperl,W. 272 Spraggins, Y.R. 254 Stephenson, T.J. 22 Stock, GJ. 10 Suguihara, C. 137

Tai,K,F.Y. 86 Tateishi,H. 146 Taylor, W. 76 Toppare, M. 267 Tötsch,M. 272 Toubas,P.L. 247

Tovar,A. 119 Trifiletti, R.R. 65

Valenzuela, F. 221 Vosatka,RJ. 65

Weintraub,Z. 188 Welch, E. 137 Wheeler, J.G 214 Wiberg,K. 230 Widness,J.A. 50

Widschwendter, M. 272 Wiese,B. 10 Wong,H.A. 311 Wyatt,E. 247

Xu,G.-Q. 302 Xu,R.-J. 280

Yamano, T. 302 Yamanouchi, I. 146 Yamashita, Y. 359 Yamauchi, Y. 146 Yoshimura, M. 100

Youinou,P. 175 Yoxall,B. 22

Zorzi Meneguzzo, C. 45

378

Author Index 